\title{
Semi-Supervised Learning for Electron Microscopy Image Segmentation
}

\author{
Eichi Takaya, ${ }^{1}$ Yusuke Takeichi, ${ }^{2}$ Mamiko Ozaki, ${ }^{2}$ Satoshi Kurihara ${ }^{1}$ \\ ${ }^{1}$ Graduate School of Science and Technology, Keio University, Japan \\ ${ }^{2}$ Graduate School of Science, Kobe University, Japan \\ etakaya@keio.jp, take.kapa3@gmail.com,mako_ozaki@hotmail.com, satoshi@keio.jp
}

\section{Introduction}

In the research field called connectomics, it is aimed to investigate the structure and connection of the neural system in the brain and sensory organ of the living things, and understand their functions. The method for investigating the neural structure varies depending on the scale, serial blockface scanning electron microscopy (SBF-SEM) is used when the target is sensory organs of small size organisms such as ants. In general, hundreds of consecutive images taken are reconstructed into three-dimensional.

Since the annotation is time-consuming task, automation by machine learning has been attempted in recent years. Many of the things that have been proposed so far are based on supervised learning, using a large amount of data labeled by experts in advance, and end-to-end learning with deep neural network has especially achieved significant results (Chen et al. 2016). However, in the case of using SBF-SEM, there is an outside chance of imaging due to the difficulty of hardening tiny sensory organs. And in most cases, therefore, a large amount of training data can not be prepared. Furthermore, labeling for training samples also places a heavy burden on experts.

In this paper, we propose a method of performing neuronal segmentation on all images for 3-D reconstruction from a very small number of training samples. This makes it possible to automatically segment neuronal regions using only a small amount of labeled data.

\section{Related Work}

Regarding the task of neuronal cell segmentation, various methods have been proposed with the related competition such as ISBI Challenge. In particular, since a method proposed by (Ciresan et al. 2012) achieved the high precision, most of newly proposed methods have been based on deep neural network. And recently, examples of achievements in recent years are Deep Contextual Network (Chen et al. 2016) and U-Net (Ronneberger, Fischer, and Brox 2015).

Though both of them have characteristics in terms of the network structure respectively, their networks are based on the Fully Convolutional Network (Long, Shelhamer, and Darrell 2015), and the feature maps obtained in the middle

Copyright (c) 2019, Association for the Advancement of Artificial Intelligence (www.aaai.org). All rights reserved.

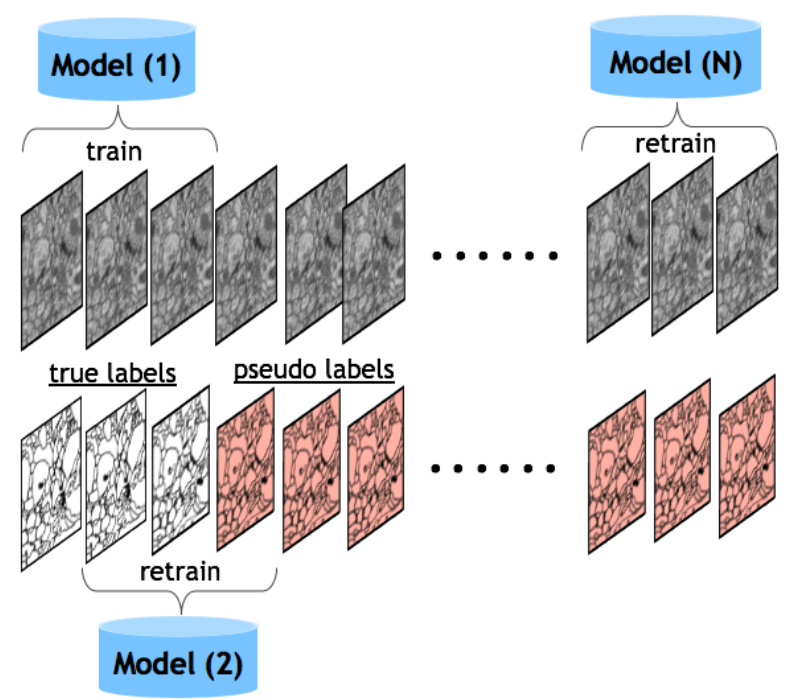

Figure 1: An overview of proposed method. Three labeled sample is used for first training, and trained model predicts a label of next sample. Then the model is retrained with next three samples to make a next pseudo label.

layers are directly leveraged by the skip connection. On the latest study, much deeper network with residual connection has been proposed to pursue further improvement (Xiao et al. 2018). However, these previous studies are based on a sufficient amount of training samples, which makes it hard to use for researchers who can not readily prepare a large amount of labeled data. Our proposed method is usable even in such cases because of leveraging a few labeled samples.

\section{Proposed Method}

The task addressed by aforementioned studies is regarded as pixel-wise classification, and according to the earlier study, it is possible even with a small amount of data (Ciresan et al. 2012). Therefore, we propose a semi-supervised learning (SSL) algorithm which learns and infer all data with pseudo labeling (Lee 2013) using a small number of consecutive training samples. An outline of the SSL algorithm is shown in Fig 1 and Algorithm 1. Firstly, supervised learning is suf- 


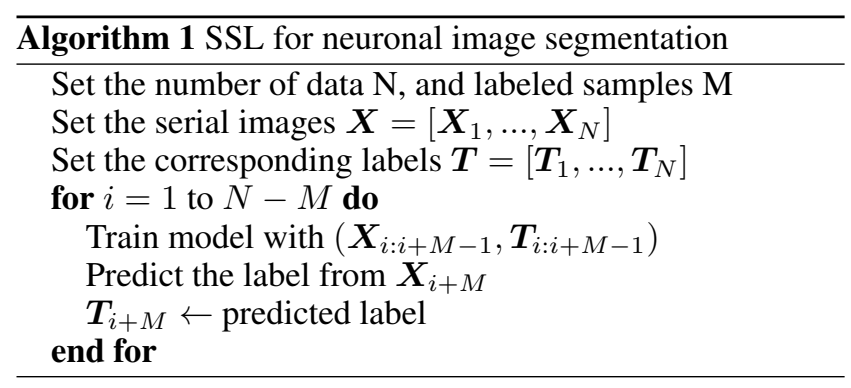

ficiently performed using a few consecutive labeled training samples. Then, the inference result for the next one image is set as a pseudo label. Next, the model is retrained with next training samples including pseudo labels. This procedure is repeated until all pseudo labels are predicted. Although what to use for the training model is arbitrary, it is desirable to use one based on Fully Convolutional Networks (Long, Shelhamer, and Darrell 2015) because of its capability of end-to-end learning.

\section{Experimental Results}

We evaluated our approach on the dataset of ISBI 2012 EM Segmentation Challenge. The training dataset consists of a stack of 30 slices. Our Performance on the 30th slice was measured by metrics Rand Score Thin $\left(V^{\text {rand }}\right)$ and Information Score Thin $\left(V^{i n f o}\right)$. The detail of their definitions is referable in (Arganda-Carreras et al. 2015) Additionally, we used another image dataset of olfactory sensory unit in the Japanese carpenter ant, taken by Ozaki laboratory in Graduate School of Science, Kobe University (Takeichi et al. 2018), and it consists of a stack of 377 slices. The performance of our inference on the 100th slice in this dataset was measured by Intersection over Union. In training process, Deep Contextual Network was used as training model. Each training phase in our SSL, we trained the model for 200 epochs with the batch size of 3. See (Chen et al. 2016) for details about the network structure and hyperparameter setting.

Table 1 and 2 show the quantitative result of our performance on two datasets respectively. Comparison model was trained with only three labeled samples without semisupervised method. Our method outperformed the comparison model on both datasets. It leads to the conclusion that our approach is effective in case we have few labeled samples.

\section{Future Work}

In our experiments, while our method was shown the effectiveness of training with a few labeled samples, the accuracy was not sufficient yet. In the future, we will consider the task characteristic that there is spatial correlation between slices. Leveraging recurrent neural networks or 3-D convolutional neural networks are likely to exert their abilities.

Also, in order to perform pseudo labeling more accurately, it will be necessary to apply some kind of postprocessing.
Table 1: Results in ISBI 2012 dataset (only the first three labels were used). The evaluation metrics were calculated on the 30th prediction

\begin{tabular}{lcc}
\hline Method & $V^{\text {rand }}$ & $V^{\text {info }}$ \\
\hline \hline Supervised learning & 0.0645 & 0.1353 \\
Semi-supervised learning & $\mathbf{0 . 7 3 1 0}$ & $\mathbf{0 . 2 1 6 7}$ \\
\hline
\end{tabular}

Table 2: Results in the dataset of olfactory sensory unit in the Japanese carpenter ant (100/377 slices and the first three labels were used). The evaluation metrics were calculated on the 100th prediction

\begin{tabular}{lc}
\hline Training method & Intersection over Union \\
\hline \hline Supervised learning & 0.3426 \\
Semi-supervised learning & $\mathbf{0 . 5 3 1 4}$ \\
\hline
\end{tabular}

\section{References}

Arganda-Carreras, I.; Turaga, S. C.; Berger, D. R.; Cireşan, D.; Giusti, A.; Gambardella, L. M.; Schmidhuber, J.; Laptev, D.; Dwivedi, S.; Buhmann, J. M.; et al. 2015. Crowdsourcing the creation of image segmentation algorithms for connectomics. Frontiers in neuroanatomy 9.

Chen, H.; Qi, X.; Cheng, J.-Z.; Heng, P.-A.; et al. 2016. Deep contextual networks for neuronal structure segmentation. In $A A A I, 1167-1173$.

Ciresan, D.; Giusti, A.; Gambardella, L. M.; and Schmidhuber, J. 2012. Deep neural networks segment neuronal membranes in electron microscopy images. In Advances in neural information processing systems, 2843-2851.

Lee, D.-H. 2013. Pseudo-label: The simple and efficient semi-supervised learning method for deep neural networks. In Workshop on Challenges in Representation Learning, ICML, volume 3, 2.

Long, J.; Shelhamer, E.; and Darrell, T. 2015. Fully convolutional networks for semantic segmentation. In Proceedings of the IEEE Conference on Computer Vision and Pattern Recognition, 3431-3440.

Ronneberger, O.; Fischer, P.; and Brox, T. 2015. U-net: Convolutional networks for biomedical image segmentation. In International Conference on Medical Image Computing and Computer-Assisted Intervention, 234-241. Springer.

Takeichi, Y.; Uebi, T.; Miyazaki, N.; Murata, K.; Yasuyama, K.; Inoue, K.; Suzaki, T.; Kubo, H.; Kajimura, N.; Takano, J.; Omori, T.; Yoshimura, R.; Endo, Y.; Hojo, M. K.; Takaya, E.; Kurihara, S.; Tatsuta, K.; Ozaki, K.; and Ozaki, M. 2018. Putative neural network within an olfactory sensory unit for nestmate and non-nestmate discrimination in the japanese carpenter ant: The ultra-structures and mathematical simulation. Frontiers in Cellular Neuroscience 12:310.

Xiao, C.; Liu, J.; Chen, X.; Han, H.; Shu, C.; and Xie, Q. 2018. Deep contextual residual network for electron microscopy image segmentation in connectomics. In Biomedical Imaging (ISBI 2018), 2018 IEEE 15th International Symposium on, 378-381. IEEE. 